\title{
Silica-based packing materials for PREP HPLC, SFC and SMB
}

D. Sanchez

Eka Chemicals AB, Separation Products, 44580 Bohus, Sweden
Silica-based packings for preparative HPLC, SFC and SMB are today manufactured with high batch to batch reproducibility and with analytical performance. Mechanical strength, loadability and chemical stability are, among others, the parameters which influence to a large extent a problem-free process and good total economy in the purification step. In this paper these properties will be described together with the manufacturing methods for spherical, totally porous particles and the chemical methods for surface modification of the particles.

rocess chromatographic purification of pharmaceuticals is gaining more ground due to recent developments in column hardware and packing materials which together lower the total purification cost. Column systems with Dynamic Axial Compression, DAC, have made it possible to pack small, efficient particles in large diameter columns with excellent efficiency and stability. The introduction of Supercritical Fluid Chromatography, SFC, and Simulated Moving Bed, SMB, have brought solvent consumption and cost to much lower levels.

Development in packing materials with unique combination of high loadability, mechanical strength and chemical stability has also in high degree contributed to even make possible difficult purifications at moderate cost.

In this paper, the manufacturing of modern silica-based packings will be described together with the important properties of these materials and the impact they have for the users.

\section{Manufacturing methods of spherical, silica-based packings}

Historically, preparative process scale liquid chromatography has been dominated by the use of irregular particles of large size, broad size distribution and low mechanical stability. This implied many restrictions in the way of using column systems packed with these particles. During the last ten years, big improvements in the design and manufacturing of silica-base particles for preparative chromatography have occurred and spherical particles of small size and with narrow particle size distribution are, by far, the most widely used in preparative chromatography. In figure 1, the SEM photos of irregular, spheroidal and spherical particles are shown.

Spherical particles are mainly manufactured by two methods:

- Sol-gel technique.

- Hydrolysis and gelling of organic silicates (Polyethoxysiloxane technique).

\section{The Sol-gel technique}

In this technique, silicic acid is polymerized to well defined spherical, non-porous primary particles, sols, which are subsequently aggregated to form the final, porous spherical particle.

It is easier to control important parameters like pore size, pore size distribution and surface area when utilizing the solgel technique.

It is also known that silica particles produced by this technique are much more mechanically and chemically stable than materials produced by other techniques.

In figure 2, the schematic production of silica particles by the sol-gel technique is represented together with the relationship between the most important parameters.

\section{Polyethoxysiloxane technique}

Here the particles are built up in two steps. Firstly by the hydrolysis of tetraetoxysilane to polyethoxysiloxane (PES). Secondly, PES is emulsified with a mixture of alcohol, water and a catalyst which initiates the polycondensation of the droplets of PES to yield the spheres of silica which 


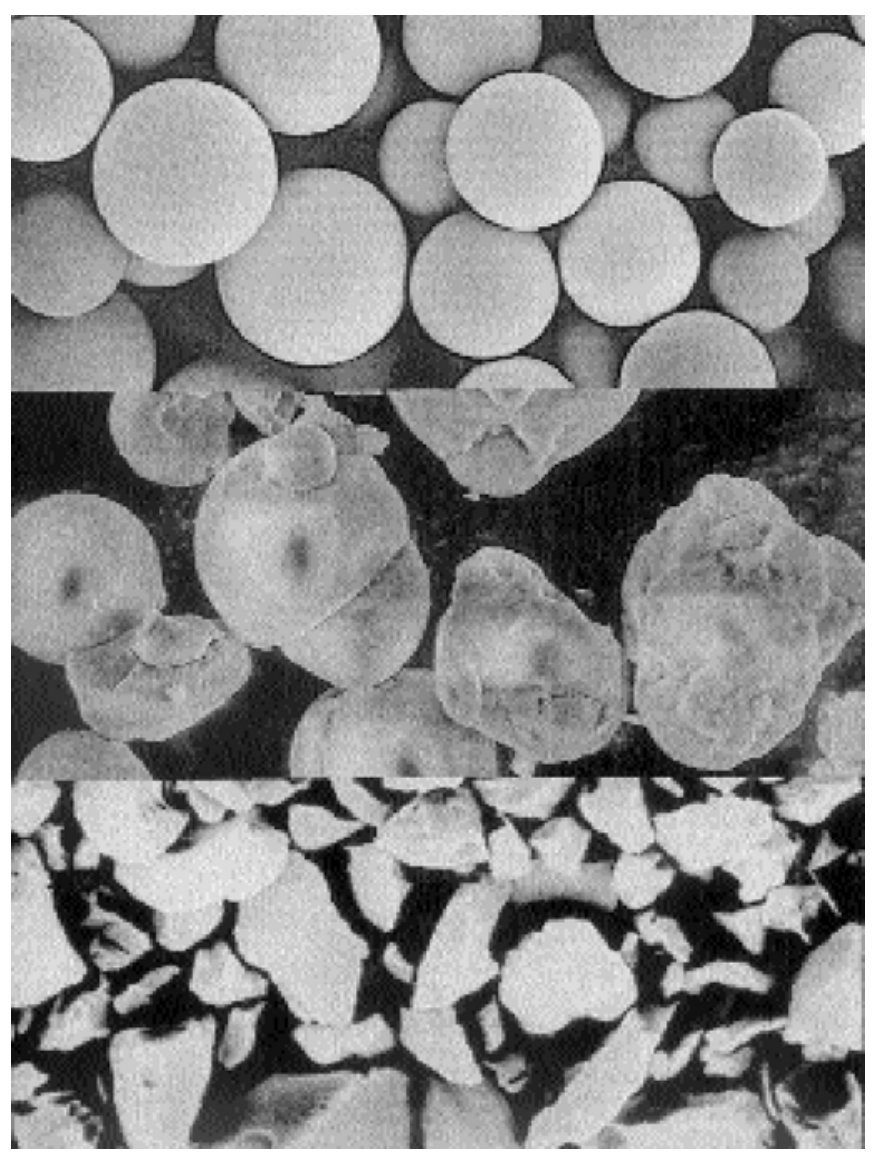

Figure 1. SEM-pictures showing spherical, spheroidal and irregular particles.

precipitate. The materials is then dried and rehydroxilated. Silica particles manufactured by this method have a spongytype porous structure which gives a broader pore size distribution and lower mechanical stability compared to the solgel technique.

\section{Important parameters of silica-based packings for preparative HPLC, SFC and SMB}

Large scale chromatography puts higher demands on packings than analytical chromatography. The main reasons are that materials have to stand the high piston pressure usually used in the systems. In addition, they have to have high loadability and chemical stability to give better total economy of the purification process.

\section{Mechanical strength}

In large bore DAC column systems, the piston pressure exerted when packing the column could be as high as 150 bar and the pressure drop developed when using these systems at high flow rate could create fines by breaking particles. The fines clog the frits, giving rise to even higher pressure drop which, at some point, stop the pumps and develop unfavourable flow profile in the systems, thus deteriorating

\section{A. Manufacture of spherical silica for HPLC.}

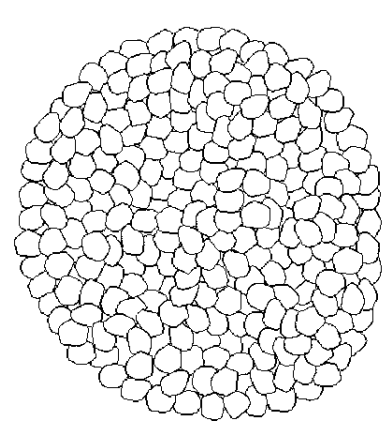

Silica sol

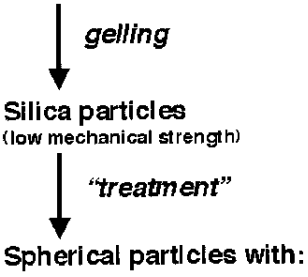

Spherical partlcles with:

- desired pore structure

- high mechanical strength

- optimized surface properties

\section{B. Manufacture of spherical silica for preparative HPLC.}

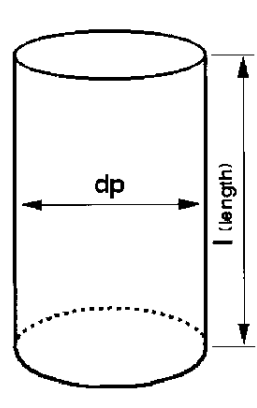

\author{
Main objective - high mechanical strength \\ - high accessible surface area \\ - optimized surface properties \\ Assume cylindrical pores \\ $\rightarrow \quad S A=\pi \cdot d p \cdot I\left(m^{2} / g\right)$ \\ $P V=\pi\left(\mathrm{dp}^{2} / 4\right) \cdot 1 \cdot 10^{6}(\mathrm{ml} / \mathrm{g})$ \\ $\rightarrow \quad P V=d p \cdot S A \cdot 10^{6} / 4$ \\ $\rightarrow \quad 40000$ PV/SA $=d p(\AA)$ \\ $\rightarrow$ high PV $\rightarrow$ high SA \\ but low mechanical strength
}

Figures 2A \& B. Scheme over the manufacturing of spherical silica according to the sol-gel technique and the relationship between the important parameters.

the performance of the column. For this reason, some manufacturers test every batch of silica for mechanical stability.

To test the mechanical stability, the particles are packed in a preparative column (50 mm ID) with a short bed $(50 \mathrm{~mm})$ and axial compression. The piston pressure is increased step by step from 50 bar all the way up to 300 bar. Pressure is then released completely and a new series of experiments is repeated. In figure 3 , the increase in bed pressure drop between two series of experiments and the particle size analysis after the performance of pressure tests of some commercially available preparative materials are shown. The lower the increase in bed pressure, the higher is the mechanical stability of the material.

\section{Loading capacity}

For preparative and process scale chromatography, loadability is probably the most important property besides selectivity.

Loadability is determined by the following parameters: Surface Area (SA), Pore Size (PD) and Size Distribution $\left(\mathrm{dp}_{90 / 10}\right)$, and in some cases (e.g. chiral purifications), Ligand Density (LD). When these parameters are optimized, High Available Surface Area/Unit Column Volume is obtained. 
Step by step increase of piston pressure (50/300 bar)

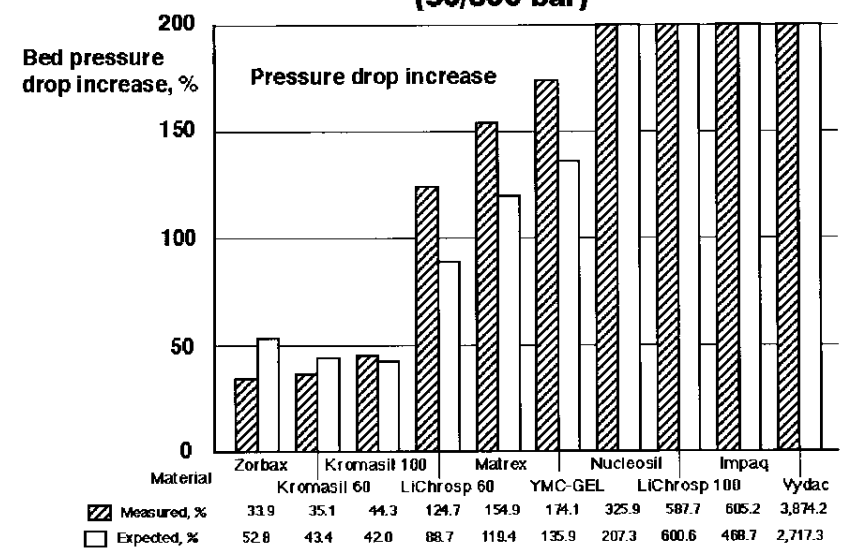

Figure 3A. Pressure drop increase after one compression cycle for Kromasil and some other commercial materials. Materials showing pressure drop higher than that expected from porosity measurements, crash during the test. The four materials to the right show a very high pressure drop increase, out of the range.

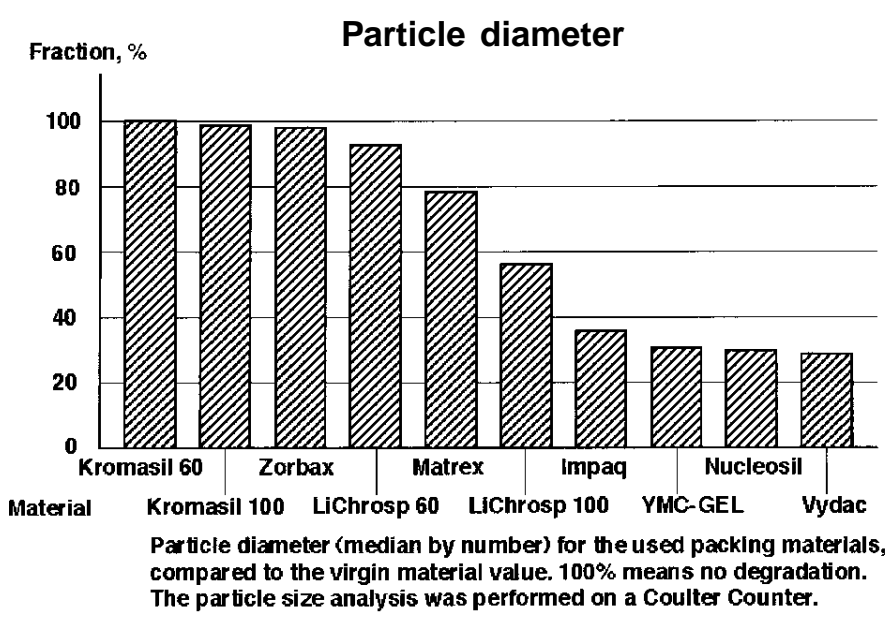

Figure 3B. Particle diameter (median by number) for the used packing materials, compared to the virgin material value. $100 \%$ means no degradation.The particle size analysis was performed on a Coulter Counter.

There is always an optimum pore size which depends on the size of the solute molecule to be purified. The relationship between the above mentioned parameters, is shown in figure 4. In figure 5, the available surface areas, SA, of Kromasil $60 \AA$ and $100 \AA$ is compared with some other commercial materials.

\section{Chemical stability}

Some silica-based materials may be sensible to hydrolysis and dissolution under respectively low and high $\mathrm{pH}$ conditions.

- The parameters that influence hydrolysis of bonded phases at low $\mathrm{pH}$ conditions are:

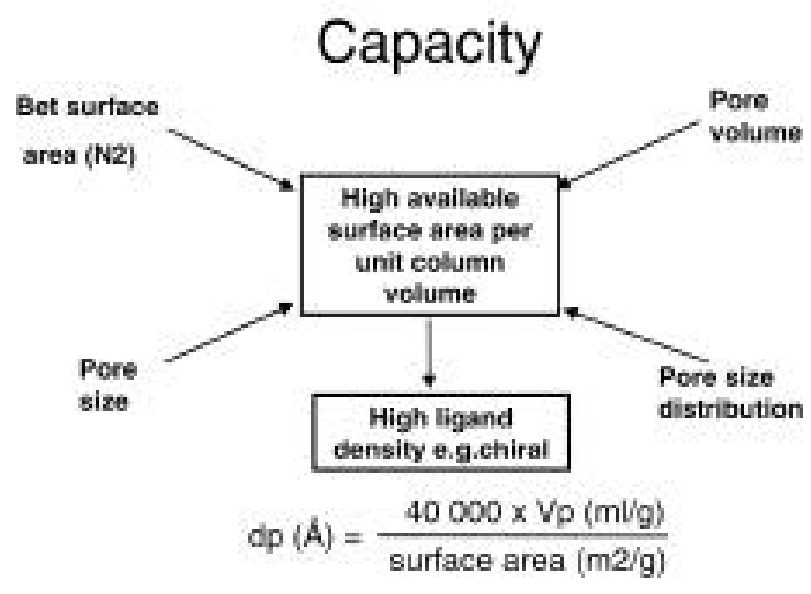

Figure 4. Important parameters influencing the available surface area of silica materials and the mathematical relationships between these.

\section{Pore size distribution}

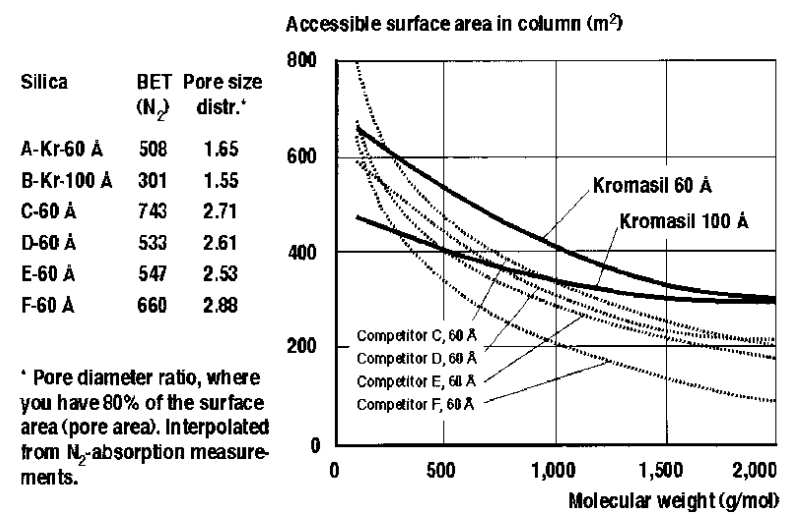

Figure 5. Available surface area of Kromasil $60 \AA$ and $100 \AA$ compared with other commercial materials of $60 \AA$ pores.

- Surface silanols: the surface of a good silica packing consists of uniformly distributed, mild type of silanol groups. The number of unwanted acidic silanols is minimized.

- Type of silane molecule used for bonding: in chemically modified silica, longer chain silanes give higher chemical stability than shorter ones, due to the fact that they protect the siloxane bonds in a more efficient way.

- Surface coverage: high surface coverage gives higher chemical stability. In this respect it is interesting to remember that modification of the silica surface with monofunctional silanes gives higher surface coverage and reproducibility than polyfunctional silanes.

- The parameters which influence dissolution of silica material under high $\mathrm{pH}$ conditions are:

- The manufacturing method: materials produced by the sol-gel technique are more stable than "spongy" ones which 


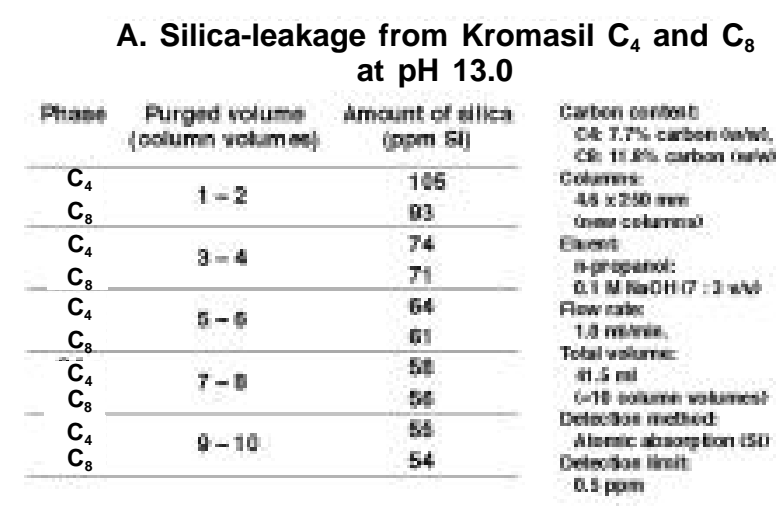

B. Silica-leakage from Kromasil $\mathrm{C}_{4}$ and $\mathrm{C}_{8}$ at $\mathrm{pH} 13.0$

\begin{tabular}{|c|c|c|c|}
\hline Phare & $\begin{array}{l}\text { Purged volume } \\
\text { [eolumn volum }\end{array}$ & $\begin{array}{l}\text { Amount of silles } \\
\text { (pDm S) }\end{array}$ & 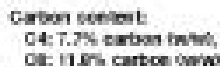 \\
\hline \multirow{2}{*}{$\begin{array}{r}-\mathrm{C}_{4} \\
\mathrm{C}_{8}\end{array}$} & \multirow{2}{*}{$1-2$} & 106 & \multirow{10}{*}{ 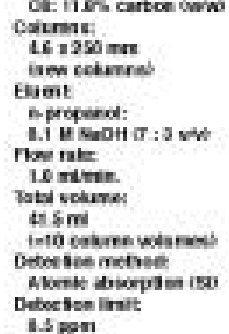 } \\
\hline & & os & \\
\hline \multirow{2}{*}{$\begin{array}{r}C_{4} \\
-\quad C_{8}\end{array}$} & \multirow{2}{*}{$3=4$} & 74 & \\
\hline & & $\pi t$ & \\
\hline $\mathrm{C}_{4}$ & \multirow{2}{*}{$5-8$} & 64 & \\
\hline $\mathrm{C}_{8}$ & & 61 & \\
\hline $\mathrm{C}_{4}$ & \multirow{2}{*}{$7-8$} & 50 & \\
\hline $\mathrm{C}_{8}^{4}$ & & Es & \\
\hline \multirow{2}{*}{$\begin{array}{l}\mathrm{C}_{4} \\
\mathrm{C}_{8}\end{array}$} & \multirow{2}{*}{$\theta=10$} & 56 & \\
\hline & & 54 & \\
\hline
\end{tabular}

C. Silica-leakage from Kromasil $\mathrm{C}_{4}$ and $\mathrm{C}_{8}$ at $\mathrm{pH} \mathbf{1 3 . 0}$

\begin{tabular}{|c|c|c|c|}
\hline Pasae & $\begin{array}{l}\text { Purged vol ime } \\
\text { \{echimen volumes; }\end{array}$ & $\begin{array}{l}\text { Amoum of alita } \\
\text { [ppn S: }\end{array}$ & 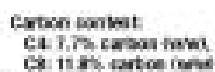 \\
\hline $\begin{array}{l}\mathrm{C}_{4} \\
\mathrm{C}_{8}\end{array}$ & $1-2$ & $\begin{array}{l}106 \\
03\end{array}$ & 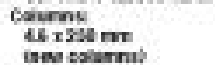 \\
\hline $\mathrm{C}_{4}$ & $3-4$ & 74 & Dumt \\
\hline $\mathrm{C}_{8}$ & $3-4$ & 71 & 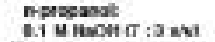 \\
\hline $\begin{array}{l}\mathrm{C}_{4} \\
\mathrm{C}_{8}\end{array}$ & $5=6$ & 64 & 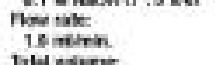 \\
\hline $\begin{array}{l}\mathrm{C}_{4} \\
\mathrm{C}_{8}\end{array}$ & $7=0$ & $\begin{array}{l}58 \\
G 8\end{array}$ & 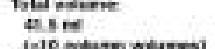 \\
\hline $\begin{array}{l}\mathrm{C}_{8} \\
\mathrm{C}_{4}\end{array}$ & $9-10$ & $\begin{array}{l}65 \\
54\end{array}$ & 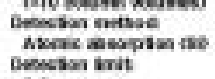 \\
\hline
\end{tabular}

Figures 6 A, B \& C. Leakage of bonded phases and silica from one commercial material, Kromasil, at different mobile phase conditions.

are produced by reacting organo-silicates under basic conditions and gelling the resulting silicic acid in situ.

- Surface treatment of the silica: the methods are numerous and generally patented.

- Coating density of the silane: see the previous comments under "Stability at low $\mathrm{pH}$ conditions".

The advantages of using chemically stable silica-based materials are clear:

- Longer lifetime which gives better total economy.

- No changes in retention times and adsorption characteristics (reproducibility).

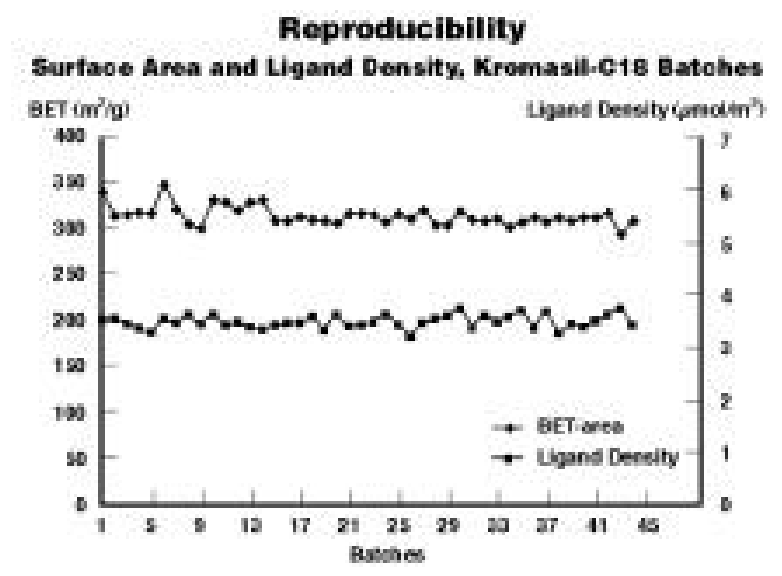

Figure 7. Batch to batch reproducibility of 50 batches of Kromasil $C_{18}$ with respect of $k^{\prime}$ and $\alpha$.

\section{Modification Procedures of Silica Gels}

a) With monofunctional silanes

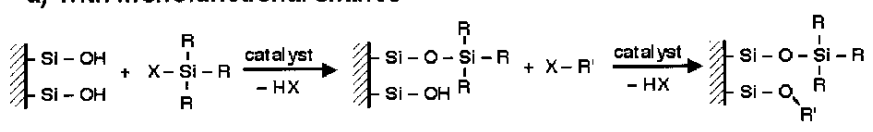

b) With polytunctional silanes

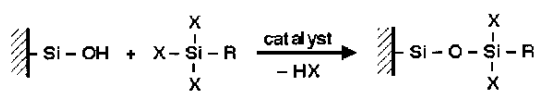

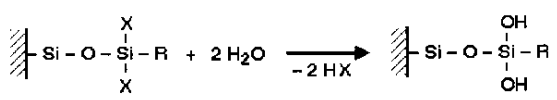

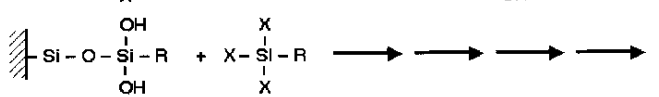

Figure 8. Scheme showing two of the most used methods for surface modification of silica surface for RP-HPLC.

- Minimized contamination of the purified product by leakage from the silica matrix or the silane ligands.

In figure 6 the low leakage degree of bonded phases and silica from one commercial material, Kromasil ${ }^{\circledR}$ is shown.

\section{Surface modification of silica materials for HPLC, SFC and SMB}

Silica materials for preparative chromatography are used unmodified for normal phase chromatography and surface modified for reversed phase, chiral or ion exchange chromatography.

The surface modification of silica for chromatography is almost always achieved by reacting the surface silanols of the silica with organic silanes. Best batch to batch reproducibility is obtained by using mono functional silanes. This is illustrated in figure 7 for the surface modification of Kromasil $^{\circledR} 100 \AA$ silica with a $\mathrm{C}_{18}$ silane. The chemical 


\section{Synthesis of the chiral stationary phases}

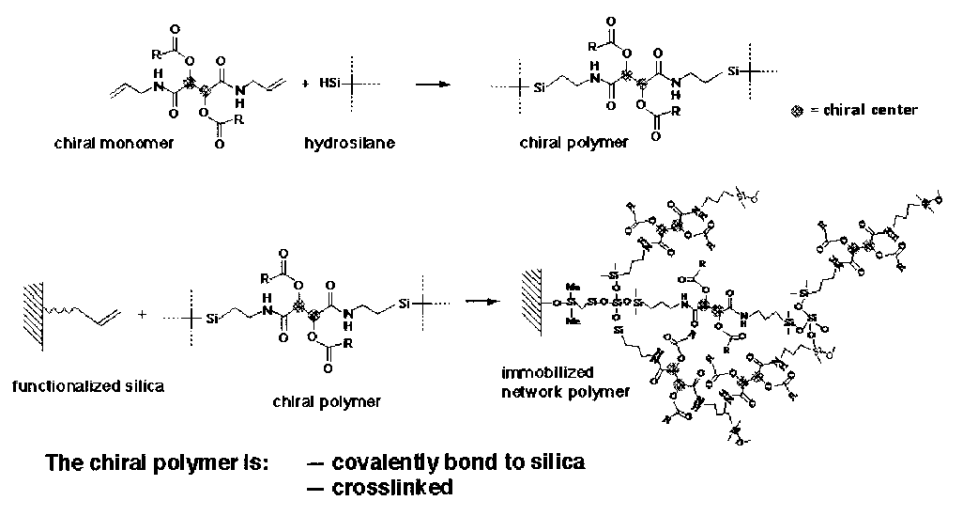

Figure 9. Shows the modification of a silica surface with covalently bound and crosslinked chiral polymer from tartaric acid diamid (DATD) derivatives.

\section{Regeneration of Kromasil at high pH}

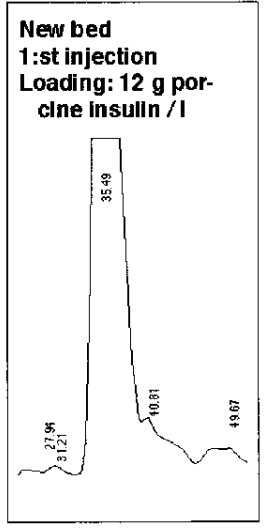

Backpressure: 35 bar

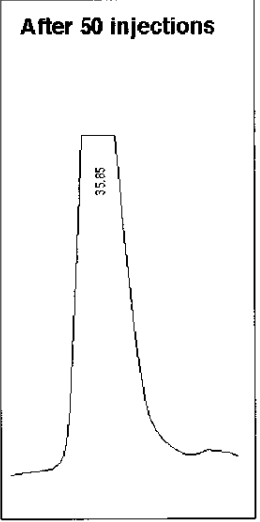

Backpressure: 65 bar

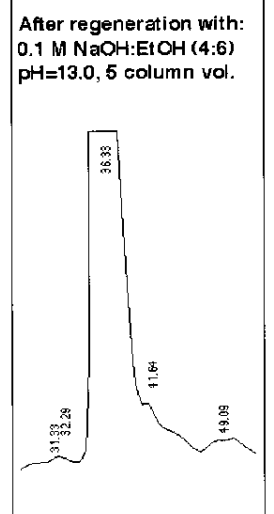

Backpressure: 35 bar

Figure 10. Regeneration of a Kromasil RP-column contaminated with polymeric insulin, at $\mathrm{pH}>13$.

modification of silica for reversed phase chromatography is shown in figure 8 . It illustrates general modification methods with monofunctional and polyfunctional silanes.

Even very advanced modifications of silica gels surfaces can be achieved today as shown for the modification of Kromasil silica with a chiral polymer (see Fig. 9).

How the silica surface is modified, determines the chemical stability (to hydrolysis and dissolution) of the packing materials, as mentioned before. In order to illustrate the chemical stability of modern silica-based packings, the regeneration of an industrial scale column used for the purification of insulin is shown in figure 10 . 